\title{
Microstructural and mechanical characterization of cement pastes subjected to static magnetic fields
}

\author{
J. A. Ortiz-Lozano ${ }^{1}$, J. J. Soto-Bernal ${ }^{2}$, R. Gonzalez-Mota ${ }^{2}$ \\ \& I. Rosales-Candelas ${ }^{2}$ \\ ${ }^{I}$ Department of Construction and Structures, \\ Autonomous University of Aguascalientes, Mexico \\ ${ }^{2}$ Department of Electronic Engineering, \\ Instituto Tecnologico de Aguascalientes, Mexico
}

\begin{abstract}
This work presents the results of an experimental study carried out to comprehend the physical, mechanical and microstructural behavior of cement pastes subjected to static magnetic fields while hydrating and setting. The experimental methodology consisted of exposing fresh cement pastes to static magnetic fields at three different magnetic induction strengths: 19.07, 22.22 and 25.37 Gauss. The microstructural characterization makes evident that there are differences in relation to amount and morphology of $\mathrm{CSH}$ gel; the amount of $\mathrm{CSH}$ is larger and its morphology becomes denser and less porous with higher magnetostatic induction strengths, also shows the evidence of changes in the mineralogical composition of the hydrated cement pastes. The temperature increasing has no negative effects over the cement paste compressive strength since the magnetostatic field affects the process of hydration through a molecular restructuring process, which leads to cement pastes being improved microstructurally, with a reduced porosity and a higher mechanical strength.

Keywords: magnetic fields, Portland cement paste, cement hydration, compressive strength, microstructural characterization.
\end{abstract}

\section{Introduction}

Cement can be described as a material with both adhesive and cohesive properties, which gives it the ability to agglutinate mineral fragments. The 
methods or techniques that increase the adhesive and cohesive properties are sought continuously. A review of the theoretical and experimental studies related to hydration of cement and microstructure of hardened cement paste, mortar and concrete is presented by Stark [1].

It is well known that the water presence and water transport both play key roles in determining the pore structure and mechanical strength as well as long term durability of cementitious materials. Magnetic treatment of water has been carried out in many studies to improve the properties of cementitious materials [2-6]. On the other hand, a magnetic field can accelerate the crystallization of sparingly soluble diamagnetic salts of weak acids like calcium carbonate [7] and gypsum [8]. There is a marked effect of magnetic field on the crystallization of calcium carbonate in ordinary water to $0.19 \mathrm{~T}$ [7] and an alignment of micronsized crystals dispersed in fluid at room temperature, this is achieved below $2 \mathrm{~T}$ for gypsum [8]. Farrell [9] reported that concrete acquires natural remanent magnetization, due to the earth's magnetic field, at casting and presented measurements of the direction and intensity of magnetization in concrete samples with and without fly ash.

Four compounds are considered as the main components of Portland clinker: tricalcium silicate $\left(3 \mathrm{CaO} \cdot \mathrm{SiO}_{2}\left(\mathrm{C}_{3} \mathrm{~S}\right)\right)$, dicalcium silicate $\left(2 \mathrm{CaO} \cdot \mathrm{SiO}_{2}\left(\mathrm{C}_{2} \mathrm{~S}\right)\right)$, tricalcium aluminate $\left(3 \mathrm{CaO} \cdot \mathrm{Al}_{2} \mathrm{O}_{3} \quad\left(\mathrm{C}_{3} \mathrm{~A}\right)\right)$ and aluminoferrite tetracalcium $\left(4 \mathrm{CaO} \cdot \mathrm{Al}_{2} \mathrm{O}_{3} \cdot \mathrm{Fe}_{2} \mathrm{O}_{3}\left(\mathrm{C}_{4} \mathrm{AF}\right)\right)$. When Portland cement is mixed with water, its four crystallographic phases start to hydrate. The reaction of tricalcium aluminate $\left(\mathrm{C}_{3} \mathrm{~A}\right)$ and ferrite phase $\left(\mathrm{C}_{4} \mathrm{AF}\right)$ predominates at early ages of hydration. The reaction of calcium silicate phases $\left(\mathrm{C}_{3} \mathrm{~S}\right.$ and $\left.\mathrm{b}-\mathrm{C}_{2} \mathrm{~S}\right)$ predominates from about the time of initial set going onward, forming calcium silicate hydrates and $\mathrm{Ca}(\mathrm{OH})_{2}[10,11]$.

Hydration is a heterogeneous process where there are at least two solid phases concerned; the initial (anhydrous) and final (hydrated) phases, and one liquid phase. According to the model documented by Taylor [12], the mechanism of hydration is based on the effect of saturation/supersaturation of $\mathrm{Ca}^{2+}$ ion on the hydration of $\mathrm{C}_{3} \mathrm{~S}$ particles, via assisting a morphological change from an impervious calcium silicate.

The effects of the treatment of fresh cement pastes with $\mathrm{CO}_{2}$ laser radiation $(10.6 \mu \mathrm{m})$, in order to improve its mechanical properties in addition to obtaining lower setting times than those of a natural setting (without radiation) were reported before [13]. Also, the changes occurred in cement pastes irradiated by $10.6 \mu \mathrm{m} \mathrm{CO} \mathrm{CO}_{2}$ laser at different stages of hydration after preparation by using Raman spectroscopy, X-ray diffraction and Scanning Electronic Microscopy (SEM) techniques used to observe molecular structural changes were presented [14].

Furthermore, the effects of a magnetic field on diamagnetic materials like water, have been considered to occur by means of the magnetic orientation; influences of magnetic field on microscopic structures and macroscopic properties of water have been studied by the spectrum techniques of infrared, Raman, visible, ultraviolet lights and X-ray [15]; Toledo et al. [16] have studied the influence of the magnetic field on physical-chemical properties of 
the liquid water. Magnetic field effects on liquid water have been studied from the Monte Carlo simulation in a lower magnetic field $(0.2 \mathrm{~T})$, also, the effect of an external magnetic field was reported on the internal energy and heat capacity of water [17].

In biochemical systems, the Larmor effect may provide a mechanism by which biological systems become sensitive to small static and alternating magnetic fields [18]. These direct effects of electromagnetic fields on single particles have been extensively studied in the classical case of the action of Lorentz's force on ions (e.g. $\mathrm{Ca}^{2+}$ ) [19]. The Lorentz model characterizes magnetic field effects on binding and transport of charge carriers via the magnetic Lorentz force. For a charged particle under viscous forces and a magnetic field, the equation of motion is [20]:

$$
m \frac{d^{2} x}{d t^{2}}+m \beta \frac{d x}{d t}+q \frac{d x}{d t} \times B+F_{\text {end }}+F_{\text {noise }}=0,
$$

where $\mathrm{x}$ is the position vector; $\mathrm{m}$ is the particle mass; $\beta$ is the viscous damping (due to thermal collisions); $\mathrm{q}$ is the particle charge; $\mathrm{B}$ is the magnetic field vector; Fend is the force due to binding site geometry and/or endogenous intermolecular forces and Fnoise is the force due to thermal noise. The Lorentz model yields a lower limit in the mT-range (10-100 Gauss) for static magnetic field effects on ion binding kinetics [20].

Given a static magnetic field oriented along the z-axis, the Lorentz-Langevin equation may be written as the following $[19,21]$ :

$$
\frac{d^{2} r}{d t^{2}}=-\beta \frac{d r}{d t}+\gamma \frac{d r}{d t} \times B k-\omega^{2} r+n
$$

where $r$ is the position vector of the particle, $\gamma$ is the ion charge-to-mass ratio $(\mathrm{q} / \mathrm{m}), \mathrm{k}$ is the unit vector along the $\mathrm{z}$-axis, $\mathrm{w}$ is the angular frequency of the oscillator, and $n$ is the random thermal noise force per unit mass. The Lorentz equation yields a coherent solution describing precession of the bound-ion oscillator orientation at the Larmor frequency in an exogenous magnetic field [21].

$$
\omega_{L}=\frac{\gamma B}{2},
$$

This paper presents the results of an experimental study carried out to comprehend the physical, mechanical and microstructural behavior of cement pastes under the influence of static magnetic fields while setting is taking place.

\section{Experimental methodology}

\subsection{Materials}

The cement pastes were prepared with a composite Portland cement classified as CPC 30 R pursuant to Mexican standard NMX-C-414-ONNCCE-2004 [22], with a 7-day compressive strength of $30 \mathrm{MPa}$, this is one of the most commonly used commercial cements in Mexico. 


\subsection{Mixture of fresh cement pastes and environmental conditions}

The cement pastes were mixed as specified in standard ASTM C 305 [23] with a water/cement ratio of 0.50 . This $\mathrm{W} / \mathrm{C}$ ratio was chosen because it is the value specified for determining normal cement paste consistency in standard ASTM C 187 [24]. The hardened cement paste samples consisted in 75 x $50 \mathrm{~mm}$ cylindrical specimens. The experimental tests were carried out in a closed laboratory in which the environmental conditions (temperature and relative humidity) were controlled and were registered consistently; the environmental temperature during the tests ranged between 18 and $22^{\circ} \mathrm{C}$.

\subsection{Exposure of the specimens to static magnetic fields}

The experimental methodology consisted in assessing the fresh cement pastes setting times as well as compressive strength at respectively, 24 hours, 3 days and 7 days of hardened cement paste samples. Such samples were exposed to a treatment of static magnetic fields at three different magnetic induction strengths immediately after being mixed, during 24 hours. Later on, the samples were stored for curing in wet environmental conditions: temperature of $20^{\circ} \mathrm{C}$ and relative humidity higher than $95 \%$.

Alongside, some other cement pastes samples were mixed and casted without having been subjected to static magnetic fields, in order to take them as comparative reference. In the case of microstructural characterization by means of Raman Spectroscopy, some samples were characterized after 24 hours after they have been mixed and others were treated 7 days after they hardened, in order to test out that magnetization posterior to that time length has no effects on hardened cement paste microstructure.

A magneto static field generator (MFG) was made in order to obtain magnetic field strengths of 0 to 30 Gauss in the exposure zone (EZ). The MFG consists of a ferromagnetic rectangular core (iron) $(25 \times 10 \mathrm{~cm})$, surrounded by a coil (insulated solid copper wire of 400 turns), with a current intensity of $3 \mathrm{~A}$, and voltage from 0 to $30 \mathrm{~V}$. The magnetic field strengths $(\mathrm{Bz})$ was measured every $1 \mathrm{~cm}$ along the Z-axial direction by using a gauss meter (F. W. Bell, Model 5180).

\subsection{Characterization tests of fresh cement paste samples}

Setting times were monitored in all the cement pastes samples with a Vicat apparatus, according to standard ASTM C 191 [25].

\subsection{Characterization tests of hardened cement paste samples}

\subsubsection{Compressive strength}

Five 75 x $50 \mathrm{~mm}$ cylindrical specimens were prepared for each magnetic field strength (B) in order to test them for compressive strength at 24 hours, 3 and 7 days. 


\subsubsection{Microstructure}

Magnetic treated samples were characterized by Raman spectroscopy and Scanning Electronic Microscopy (SEM). Profile intensity changes were recorded in the Raman spectra of the cement paste samples after magnetic treatment. Raman spectra were taken with a Renishaw model 1000 Raman spectrometer (excitation wavelength of $830 \mathrm{~nm}$ ) for all samples. Several spectra (up to 5) were taken and averaged for samples of a given laser treatment and age. SEM micrographs were taken from the surface of the cement paste samples after magnetic treatment with a XL30 SEM equipped with an EDAX Spectrometer for $\mathrm{X}$-ray dispersion elemental analysis.

\section{Results and analysis}

\subsection{Setting times}

Setting times results are presented next in Figure 1, for all of the samples of cement paste.

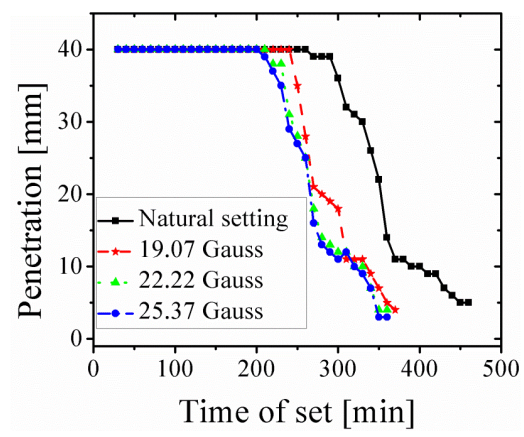

Figure 1: Setting times for the cement pastes.

The setting curves show the inverse relationship between the magnetic induction strength and the setting times, that is to say, the higher the magnetic induction strength, the faster the cement pastes set. These trends agree with the temperature evolution curves presented before.

When cement is in contact with water, $\mathrm{C}_{3} \mathrm{~S}$ dissolves [26], $\mathrm{Ca}^{2+}$ ions go into the solution and then adsorbs it at the surface, so the cement becomes positively charged [10], and calcium, silicate and hydroxide concentrations increase in solution, decreasing the degree of undersaturation and therefore the rate. The reaction of tricalcium aluminate $\left(\mathrm{C}_{3} \mathrm{~A}\right)$ and ferrite phase $\left(\mathrm{C}_{4} \mathrm{AF}\right)$ predominates at early stages of hydration. The reaction of calcium silicate phases $\left(\mathrm{C}_{3} \mathrm{~S}\right.$ and $\mathrm{b}-$ $\mathrm{C}_{2} \mathrm{~S}$ ) predominate from about the time of initial set going onward, forming calcium silicate hydrates and $\mathrm{Ca}(\mathrm{OH})_{2}$ molecules, whose are arranged around functional groups of the binder. If the magnetostatic treatment affects the cement rate of hydration, accelerating it and also the cement heat of hydration, then 
temperature, the rate of initial hydration of cement phases and the rate of formation of hydration products are increased as well.

\subsection{Compressive strength}

The compressive strength results are presented in Figure 2.

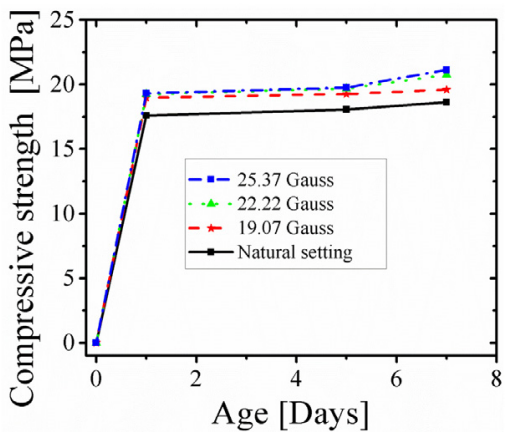

Figure 2: Compressive strength of cement pastes for each magnetic induction strength.

We can acknowledge the favorable effect of magnetic induction over the compressive strength values of the cement pastes, obtaining the higher values with the higher magnetic induction strengths. For example, the compressive strength at 7 days of the cement paste treated with 25.37 Gauss increased its compressive strength of $13 \%$ in relation to the natural setting paste, having passed from 18.6 to $21.1 \mathrm{MPa}$. Although this difference could be relative, considering the uncertainty of measurements, an increment in mechanical strength is evident.

It is well known that an increase in the early curing temperature makes the hydration rate and cement strength increase rapidly, but due to the nonhomogeneous diffusion of the hydration products the porosity of the cement paste increases and microcracks develop, which finally leads to decreased strength at a later time [27].

Although a higher temperature during placing and setting increases strength in the very early stages, it may adversely affect the strength from about 7 days going onwards. The explanation for this is that rapid initial hydration appears to form products of a poorer physical structure, which are probably more porous, so a proportion of the pores always remain unfilled. It follows from the gel / space ratio rule that this would lead to a lower strength than that of a less porous but slowly hydrating cement paste, in which a high gel/space ratio would eventually be reached.

This explanation of the adverse effects of a high early temperature on later strengths has been extended by Verbeck and Helmuth [28], who suggest that a rapid initial rate of hydration at higher temperatures slows hydration down and produces a non-uniform distribution of the products of hydration within the 
paste. The reason for this is that, at the high initial rate of hydration, there is insufficient time for the diffusion of the products of hydration away from the cement particles and for a uniform precipitation in the interstitial space (as it is the case at lower temperatures). As a result, a high concentration of the products of hydration builds up in the vicinity of the hydrating particles, and this subsequently slows hydration down and has an adverse effect on long-term strength. In addition, the non-uniform distribution of the products of hydration adversely affects the strength per se, because the gel / space ratio is lower than it would otherwise be the case for an equal degree of hydration, and the weaker local areas lower the overall strength of the hydrated cement paste [29].

Nevertheless, it can be seen that in this case, the temperature increasing has no negative effects over the cement paste compressive strength since the magnetostatic field affects the process of hydration through a molecular restructuring process, which leads to microstructurally improved cement pastes, with a reduced porosity and a higher mechanical strength.

\subsection{Microstructure}

\subsubsection{Raman spectroscopy}

The results corresponding to microstructural characterization by means of Raman spectra are presented in Figures 3 a) and b), for the cement pastes treated with magnetic induction. As stated in the experimental methodology section, the samples were characterized after 24 hours and 7 days.

Changes can be observed in the main compounds in magnetized cement pastes. The Al-O bond (Aluminum and Oxygen) can be located at $790 \mathrm{~cm}^{-1}$ peak, the peak located in the wavenumber of $987 \mathrm{~cm}^{-1}$ corresponds with the tricalcium silicate $\left(3 \mathrm{CaO} \bullet \mathrm{SiO}_{2} \bullet 8 \mathrm{H}_{2} \mathrm{O}\right)$, which is derived from the alite, main constituent of Portland cement and responsible of early age strength. In the wavenumber of $1078 \mathrm{~cm}^{-1}$ we can identify the creation of dicalcium silicate $\left(2 \mathrm{CaO} \cdot \mathrm{SiO}_{2}\right)$ which is known as belite, also a Portland cement main constituent and responsible for the long term mechanical strength, as well as the gypsum $\left(\mathrm{CaSO}_{4} \cdot 2 \mathrm{H}_{2} \mathrm{O}\right)$ which is located in the $1279 \mathrm{~cm}^{-1}$ peak. Finally the tricalcium aluminate $\left(3 \mathrm{CaO} \cdot \mathrm{Al}_{2} \mathrm{O}_{3}\right)$, also main constituent of Portland cement and in charge of instant set and very early strength, is located in the peaks of wavenumber of $1581 \mathrm{~cm}^{-1}$ and $1732 \mathrm{~cm}^{-1}$ [14].

Regarding the identification of the hydrated phase and carbonates [30], the Raman spectra of the C-S-H samples are not quite well resolved and contain a large number of bands from which to make structural interpretations. Fig. 3 clearly indicates that $\mathrm{C}-\mathrm{S}-\mathrm{H}$ samples exposed to magnetostatic fields are a slightly more crystalline. In cement samples it is also observed a band at 1090 $\mathrm{cm}^{-1}$ due to carbonate presence increased in intensity of the samples exposed to magnetostatic fields after 24 hours after mixing; but 7 days after hardening, no further increase in intensity was observed, suggesting that decomposition of C-S$\mathrm{H}$ was completed.

Taking into account that the fluorescence can give anomalous results, we can see that the bands attributed to main compounds in hydrated cement paste are increased or decreased due to the effect of magnetostatic induction and due to the 
effect of the strength therefore, i.e. the higher the magnetostatic induction strength, the higher the increment in creation of those compounds.

Likewise, it can be observed that after 7 days, the effect of magnetic treatment has no effect over the hardened cement paste, in which it is no longer possible to modify its microstructure morphologically. In a previous paper, it was concluded that a magnetic field accelerates the crystallization of sparingly soluble diamagnetic salts of weak acids, to which category calcium carbonate belongs [7].
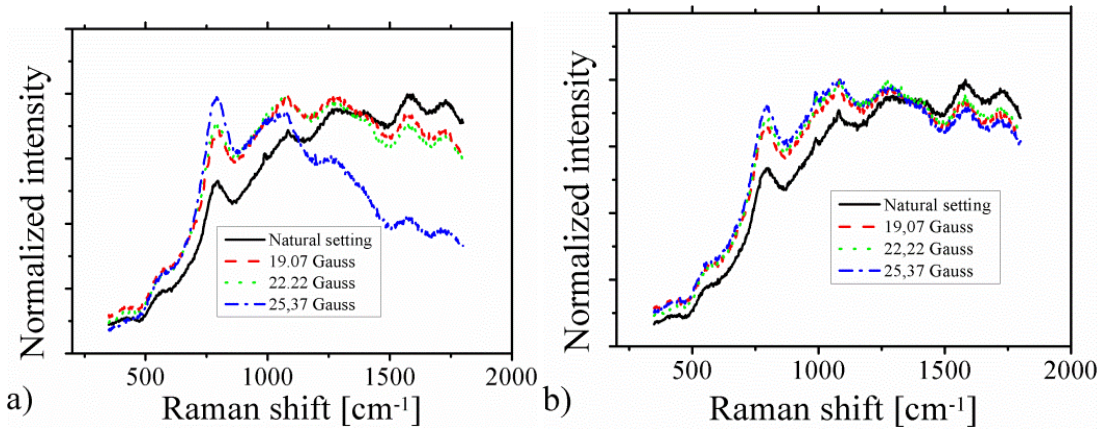

Figure 3: Raman spectra of cement pastes exposed to magnetostatic fields, a) after 24 hours after mixing and b) after 7 days after hardening.

\subsubsection{Scanning Electronic Microscopy (SEM)}

The microscopic images taken by means of SEM are presented in Figures 4 and 5. Figure 4 corresponds to the natural cement paste (without magnetic treatment) and Figures 5(a), (b) and (c), corresponding to hardened cement pastes treated with three magnetic induction strengths: 19.07, 22.22 and 25.37 Gauss, respectively.

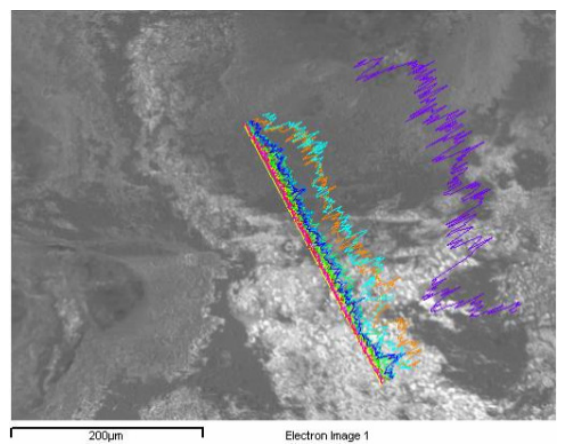

Figure 4: Appearance of cement paste sample without magnetic treatment. 
The microscopic pictures presented before demonstrate certain differences in relation to amount and morphology of CSH (Calcium Silicate Hydrate) gel, main hydration product of cement and responsible of mechanical strength development. It can be seen that the amount of CSH gel is larger and its morphology becomes denser and less porous with higher magnetostatic induction strengths; this fact favors the mechanical strength development, agreeing with the mechanical strength characterization results presented above.

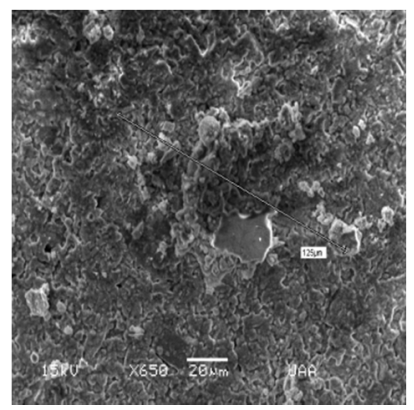

(a)

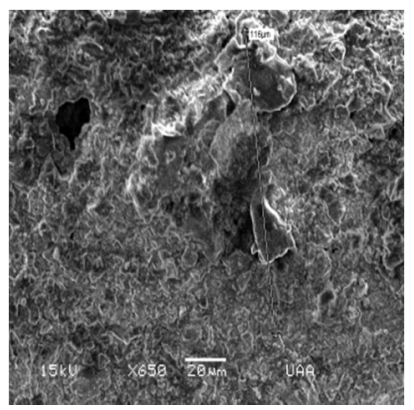

(b)

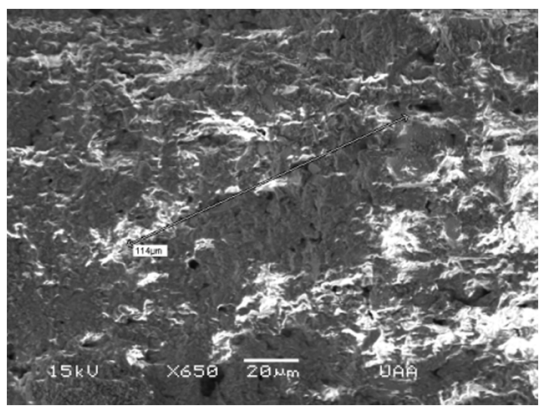

(c)

Figure 5: Appearance of cement paste samples with magnetic induction of (a) 19.07; (b) 22.22 and (c) 25. 37 Gauss.

\section{Conclusions}

Based on the experimental results, the magnetostatic treatment affects the cement rate of hydration, accelerating it and also the cement heat of hydration; also increases the temperature of cement paste samples. Regarding setting times, the higher the magnetic induction strength, the faster the cement pastes set; these trends agree with heat evolution curves.

The magnetostatic induction has favorable effects over the mechanical strength of cement paste, since the higher values of compressive strength were attained for the cement pastes treated with higher magnetic induction strengths; a relative increase of about $13 \%$ has been observed for the maximum induction strength at 7 days. 
The results corresponding to the microstructural characterization by means of Raman, show that the bands attributed to main compounds in hydrated cement paste are increased or decreased due to the effect of magnetostatic induction and due to the effect of the strength therefore, i.e. the higher the magnetostatic induction strength, the higher the increment in creation of those compounds; the effect of magnetic treatment has no any effect over the hardened cement paste after 7 days, since it is no longer possible to modify its microstructure morphologically.

The SEM pictures make evident certain differences in relation to amount and morphology of CSH gel, knowing that the amount of CSH gel is larger and its morphology becomes denser and less porous with higher magnetostatic induction strengths; this fact favors the mechanical strength development, agreeing with the mechanical strength characterization results.

It is not clear how the magnetostatic field affects the process of hydration, but a possible explanation could be related with the classical case of the action of Lorentz's force on ions, because the effect of the external magnetic field is to induce the precessional motion at the Larmor frequency in bound water molecules forming hydration layers at the binding site. The above stated can be explained since there is a great relationship among rate of hydration, heat of hydration, porosity, strength development, chemical composition and morphology of CSH gel; as well as the fact that when cement is in contact with water, $\mathrm{C}_{3} \mathrm{~S}$ dissolves, $\mathrm{Ca}^{2+}$ ions go into the solution and then are being adsorbed at the surface. Due to such mechanisms, a molecular restructuring process is created, caused by the effects of magnetostatic treatment, which leads to microstructurally improved cement pastes, with a reduced porosity and a higher mechanical strength.

\section{References}

[1] J. Stark, Recent advances in the field of cement hydration and microstructure analysis, Cem Concr Res. 41 (2011) 666-678. doi:10.1016/j.cemconres.2011.03.028.

[2] N. Su, C.-F. Wu, Effect of magnetic field treated water on mortar and concrete containing fly ash, Cem Concr Compos. 25 (2002) 681-688. doi:10.1016/S0958-9465(02)00098-7.

[3] V.V. Azharonok, N.K. Belous, S.P. Rodtsevich, V.D. Koshevar, S.V. Goncharik, N.I. Chubrik, et al., High-frequency magnetic-pulse treatment of water as a method of improving the technological properties of fine concretes, J Eng Phys Thermophys. 82 (2009) 1102-1109. doi:10.1007/s10891-010-0311-9.

[4] N.K. Belous, V.V. Azharonok, S.P. Rodtsevich, V.D. Koshevar, S.V. Goncharik, N.I. Chubrik, et al., Influence of magnetic and acoustic treatment of superplasticizer solutions on the properties of Portland cement concretes, J Eng Phys Thermophys. 85 (2012) 493-500. doi:10.1007/s10891-012-0678-x. 
[5] J. Boguszynska, M.C.A. Brown, P.J. McDonald, J. Mitchell, M. Mulheron, J. Tritt-Goc, et al., Magnetic resonance studies of cement based materials in inhomogeneous magnetic fields, Cem Concr Res. 35 (2005) 2033-2040. doi:10.1016/j.cemconres.2005.06.012.

[6] N. Su, Y.-H. Wu, C.-Y. Mar, Effect of magnetic water on the engineering properties of concrete containing granulated blast-furnace slag, Cem Concr Res. 20 (2000) 599-605. doi:10.1016/S0008-8846(00)00215-5.

[7] H.E.L. Madsen, Crystallization of calcium carbonate in magnetic field in ordinary and heavy water, J Cryst Growth. 267 (2004) 251-255. doi:10.1016/j.jcrysgro.2004.03.051.

[8] C. Uyeda, S. Kano,, K. Hisayoshi, A. Nakanishi, K. Kimoto, Efficiency of Magnetic Alignment Found in Various Paramagnetic and Diamagnetic Solids Detected by Simple Rotational Oscillation of Sample in Microgravity, Mater Trans. 48 (2007) 2893-2897.

[9] C.W. Farrell, Natural remanent magnetization of Portland Cement Concrete, Cem Concr Res. 21 (1991) 489-495. doi:10.1016/00088846(91)90098-3.

[10] M. Heikal, M.S. Morsy, Effect of treatment temperature on the early hydration characteristics of superplasticized silica fume blended cement pastes, Cem Concr Res. 35 (2005) 680-687. doi:10.1016/j.cemconres.2004.06.012.

[11] A.M. Neville, Properties of concrete, Fourth edition, Pearson Education Limited, London, 1999.

[12] H.F.W. Taylor, Cement chemistry, Second edition, Thomas Telford, London, 1997.

[13] M.R. Moreno-Virgen, J.J. Soto-Bernal, J.A. Ortiz-Lozano, A. BonillaPetriciolet, J.T. Vega-Durán, R. González-Mota, et al., Effect of CO2 laser radiation on the mechanical properties of Portland cement pastes, Mater Constr. 61 (2011) 77-91. doi:10.3989/mc.2010.54709.

[14] M.R. Moreno-Virgen, J.J. Soto-Bernal, J.A. Ortiz-Lozano, C. FraustoReyes, A. Bonilla-Petriciolet, R. González-Mota, et al., Laser radiation $\mathrm{CO} 2$ effects in cement paste at different hydration stages after preparation, Ing Investig Tecnol. XII (2011) 321-328.

[15] X.-F. Pang, B. Deng, The changes of macroscopic features and microscopic structures of water under influence of magnetic field, Phys B. 403 (2008) 3571-3577. doi:10.1016/j.physb.2008.05.032.

[16] E.J.L. Toledo, T.C. Ramalho, Z.M. Magriotis, Influence of magnetic field on physical-chemical properties of the liquid water: Insights from experimental and theoretical models, J Mol Struct. 888 (2008) 409-415. doi:10.1016/j.molstruc.2008.01.010.

[17] K.X. Zhou, G.W. Lu, Q.C. Zhou, J.H. Song, S.T. Jiang, H.R. Xia, Monte Carlo simulation of liquid water in a magnetic field, J Appl Phys. 88 (2000) 1802-1805. doi:10.1063/1.1305324.

[18] D.T. Edmonds, Larmor precession as a mechanism for the detection of static and alternating magnetic fields, Biochem Bioenerg. 30 (1993) 3-12. doi:10.1016/0302-4598(93)80057-2. 
[19] B. Bianco, A. Chiabrera, From the Langevin-Lorentz to the Zeeman model of electromagnetic effects on ligand-receptor binding, Biochem Bioenerg. 28 (1992) 355-365. doi:10.1016/0302-4598(92)80025-C.

[20] A.A. Pilla, D.J. Muehsam, M.S. Markov, A dynamical systems/Larmor precession model for weak magnetic field bioeffects: ion binding and orientation of bound water molecules, Biochem Bioenerg. 43 (1997) 239249. doi:10.1016/S0302-4598(96)05161-6.

[21] D.J. Muehsam, A.A. Pilla, Lorentz approach to static magnetic field effects on bound-ion dynamics and binding kinetics: thermal noise considerations, Bioelectromagn. 17 (1996) 89-99.

[22] S.C. Organismo Nacional de Normalización y Certificación de la Construcción y Edificación, NMX-C-414-ONNCCE Industria de la Construcción - Cementos hidráulicos - Especificaciones y métodos de prueba, Organismo Nacional de Normalización y Certificación de la Construcción y Edificación, S. C., 2004.

[23] American Society for Testing and Materials, ASTM C305-99e1 Practice for Mechanical Mixing of Hydraulic Cement Pastes and Mortars of Plastic Consistency, American Society for Testing and Materials, 2001.

[24] American Society for Testing and Materials, ASTM C187-98 Standard Test Method for Normal Consistency of Hydraulic Cement, American Society for Testing and Materials, 2001.

[25] American Society for Testing and Materials, ASTM C191-99 Standard Test Method for Time of Setting of Hydraulic Cement by Vicat Needle, American Society for Testing and Materials, 2001.

[26] K.L. Scrivener, A. Nonat, Hydration of cementitious materials, present and future, Cem Concr Res. 41 (2011) 651-665. doi:10.1016/ j.cemconres.2011.03.026.

[27] Kim J.-K., Moon Y.-H., Eo S.-H., Compressive strength development of concrete with different curing time and temperature, Cem Concr Res. 28 (1998) 1761-1773. doi:10.1016/S0008-8846(98)00164-1.

[28] J.G. Verbeck, R.H. Helmuth, Structure and Physical Properties of Cement Pastes, in: 5th Int. Congr. Chem. Cem., n.d. pp. 1-32.

[29] J. Ortiz, A. Aguado, L. Agulló, T. García, Influence of environmental temperatures on the concrete compressive strength: Simulation of hot and cold weather conditions, Cem Concr Res. 35 (2005) 1970-1979. doi:10.1016/j.cemconres.2005.01.004.

[30] M. Chollet, M. Horgnies, Analyses of the surfaces of concrete by Raman and FT-IR spectroscopies: comparative study of hardened samples after demoulding and after organic post-treatment, Surf Interface Anal. 43 (2011) 714-725. doi:10.1002/sia.3548. 